Sabrina Jahns, Lars Thorben Neustock, Moritz Paulsen, Elmira Moussavi, Martina Gerken

\title{
Plattformtechnologie für die mobile, markerfreie Proteindetektion
}

Zusammenfassung: Wir präsentieren eine Plattformtechnologie zur mobilen, markerfreien Detektion von mehreren Proteinen gleichzeitig. Ein photonischer Kristall, der mit Liganden lokal funktionalisiert ist, dient hier als Sensor. Über ein kompaktes, Kamera-basiertes Messsystem wird die Proteinanbindung an die Sensoroberfläche in ein Intensitätssignal umgewandelt, über dessen Amplitude die Proteinkonzentration bestimmt werden kann. Um das Detektionslimit dieser Technologie weiter zu verbessern, werden hier photonische Kristalle mit einer multiperiodischen und aperiodischen Gitterstruktur simulativ und experimentell untersucht. Dafür werden die Gesamtempfindlichkeit und die Resonanzgüte jeder Struktur bestimmt und mit der bisher verwendeten monoperiodischen Struktur verglichen. Es konnte festgestellt werde, dass die Resonanzgüte von mono- über multi- bis aperiodisch sich deutlich verbessert. Eine Steigerung der Gesamtempfindlichkeit konnte nicht festgestellt werden. Jedoch konnte anhand von Analysen der elektrischen Feldverteilung innerhalb der verschiedenen Strukturen beobachtet werden, dass die Modenausbreitungen in den aperiodischen Strukturen stark lokalisiert wird und die elektrische Feldintensität in diesen „Hot-Spots“ deutlich über der mittleren Feldintensität, die eine flächige Funktionalisierung repräsentiert, liegt. Diese lokale Resonanzausbildung konnte zudem bereits in ersten experimentellen Untersuchungen bestätigt werden.

Schüsselwörter: Optische Biosensoren, photonische Kristalle, multiperiodische und aperiodische Nanostrukturen, markerfrei 


\section{Einleitung}

Krankheiten wie akuter Herzinfarkt und Diabetes sollten möglichst schnell erkannt und behandelt werden, um den Krankheitsverlauf des betroffenen Patienten positiv beeinflussen zu können [1, 2]. Dafür sind kompakte, schnell arbeitende, dezentrale und einfach zu handhabende Sensoren von hohem Interesse für Point-of-Care Anwendungen. Diese Sensoren sollten in der Lage sein, spezifisch mehrere Proteine für eine eindeutige Diagnose nachzuweisen [3-5]. Im Gegensatz zu markerbasierten Messmethoden, wie z.B. der Detektion über Fluoreszenzsignale, benötigen die markerfreien Methoden keine weiteren aufwendigen Präparationsschritte der Reagenzien [6]. Generell wird bei den markerfreien Methoden über einen Signalwandler, auf dessen Oberfläche Liganden zum Fangen der zu detektierenden Proteine immobilisiert sind, die Bindung der Proteine in ein messbares Signal umgewandelt. Dieses kann elektrochemisch, kolorimetrisch oder optisch durch geführt werden [7-9]. Es wird hier eine optische Plattformtechnologie zur mobilen, markerfreien und parallelen Detektion von mehreren Proteinen auf einem Chip präsentiert. Hierfür dient ein photonischer Kristall als Signalumwandler, dessen Oberfläche lokal mit mehreren und spezifischen Liganden funktionalisiert wird, wie schematisch in Abb. 1 gezeigt [10].

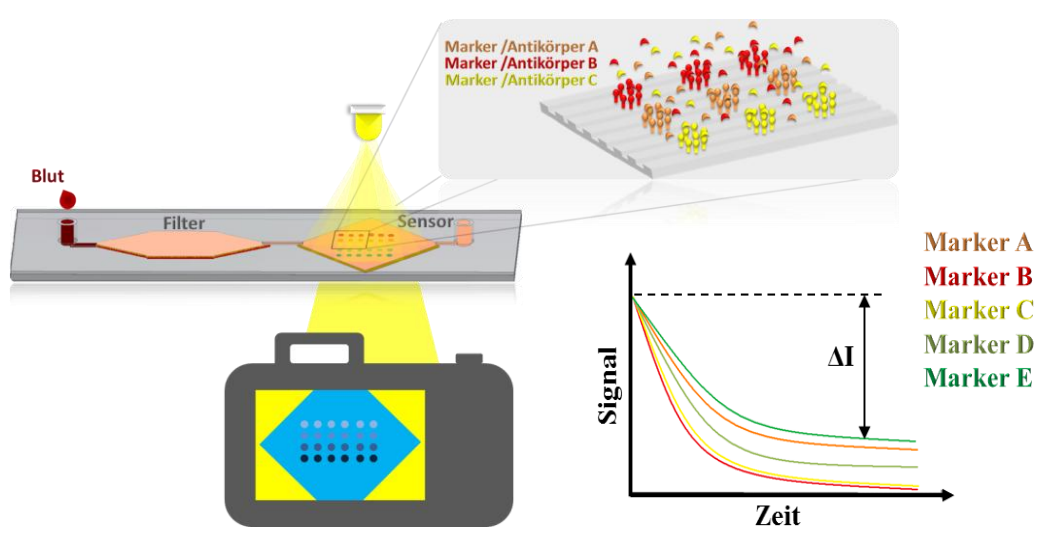

Abb. 1: Schema der Plattformtechnologie für die parallele, optische Konzentrationsmessung mehrerer Proteine in einer Blutprobe.

Ein Tropfen Blut wird auf einen Wegwerfchip gegeben und das Plasma mit einem Filter getrennt. Das Sensorfeld enthält den funktionalisierten photonischen Kristall [11].

Photonische Kristalle sind Wellenleiter mit einer periodisch nanostrukturierten Oberfläche bestehend aus einem hochbrechenden, dielektrischen Material. Dadurch können sich quasi-geführte Moden ausbilden, die sowohl im transmittierten als auch im reflektierten Lichtspektrum als Resonanzen sichtbar sind (Abb. 2). Aufgrund des evaneszenten Anteils der quasi-geführten Moden reagieren photonische Kristalle sensitiv auf Brechungsindexänderungen an der Sensoroberfläche mit einer Verschiebung der Resonanzposition.

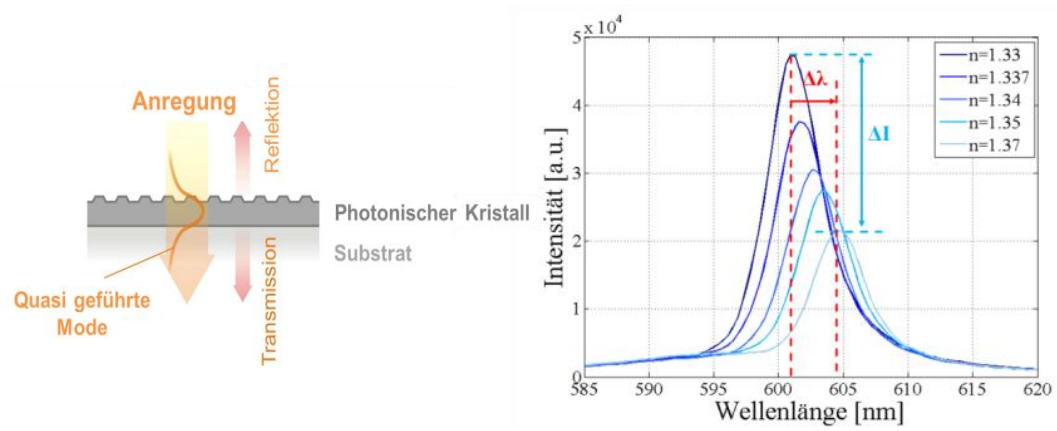

Abb. 2: Querschnitt der nanostrukturierten Oberfläche, die einen photonischen Kristall bildet, in dem quasi-geführte Moden auftreten (links). Gemessene Resonanzverschiebung $\left(\Lambda=370 \mathrm{~nm}\right.$, Tastverhältnis $\mathrm{t}=0,6$, Gittertiefe $60 \mathrm{~nm}$, Schichtdicke $\mathrm{TiO}_{2}$ von $85 \mathrm{~nm}$ ) und beleuchtungsabhängige Intensitätsänderung hervorgerufen durch Brechungsindexänderung an der Oberfläche (rechts). 
Durch ein Kamera-basiertes Messsystem wird diese Resonanzverschiebung in eine Intensitätsänderung umgewandelt, durch welche wiederum die Proteinkonzentration bestimmt werden kann. Wie aus Abb. 2 rechts entnommen werden kann, verursacht die Brechungsindexerhöhung an der Oberfläche eine Resonanzverschiebung sowie eine Intensitätsänderung. Die Intensitätsänderung wird durch die Verwendung einer farbigen LED als Anregungsquelle und die Positionierung der Resonanz an deren abfallender, spektralen Flanke verstärkt [12].

Für die optische Detektion werden die Liganden auf der 25 x $25 \mathrm{~mm}^{2}$ großen Sensoroberfläche tropfenweise kovalent gebunden. Die Bindung des passenden Proteins erzeugt an den jeweiligen Positionen eine lokale Brechungsindexänderung und somit eine Intensitätsänderung des transmittierten Lichts, die dann mit der Kamera aufgenommen wird. Mit diesem System konnten wir die parallele und spezifische Anbindung der drei Proteine CD40 Ligand Antikörper (13.5 $\mu \mathrm{g} / \mathrm{ml})$, EGF Antikörper $(13.5 \mu \mathrm{g} / \mathrm{ml})$ und Streptavidin $(30 \mu \mathrm{g} / \mathrm{ml})$ anhand von 6 Ligandpositionen nachweisen [10].
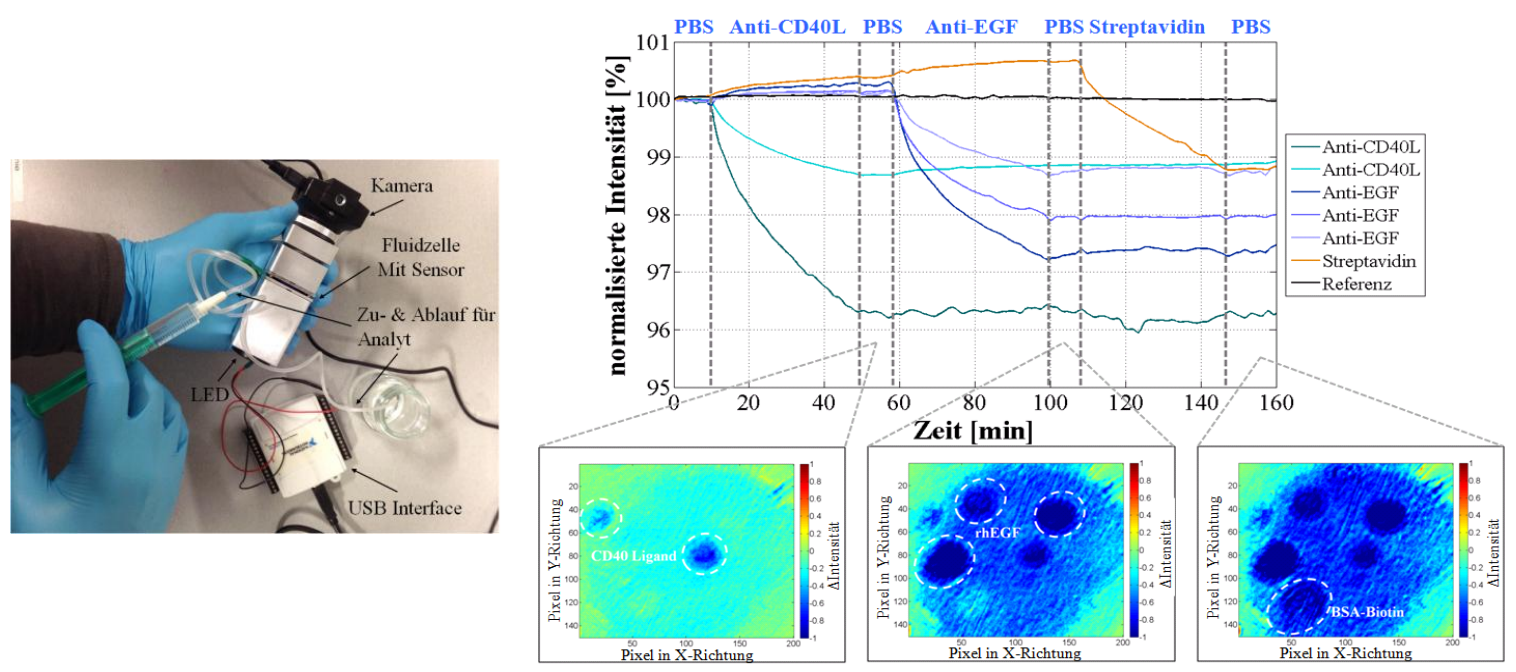

Abb. 3: Bild des Kamera-basierten Messsystems mit der Kamera, der LED, Optik innerhalb des metallischen Torsos, der Fluidzelle mit dem Sensor, Flügelkanülen für Zu- und Ablauf des Analyten und dem USB-Interface (links). Der Intensitätsverlauf und die entsprechenden Intensitätsbilder geben die Anbindungskurven bei einer Zugabe von zuerst CD40 Ligand Antikörper, dann EGF Antikörper und zum Schluss Streptavidin wieder. Es kommt zu keiner unspezifischen Bindung (rechts).

Es wird hier über zwei Verfahren zur Erhöhung der Empfindlichkeit berichtet, um das System für diagnostische Anwendungen, bei denen die relevante Proteinkonzentrationen häufig im pg/ml-Bereich liegen, attraktiver zu gestalten. Zuerst werden multiperiodische und aperiodische Nanostrukturen betrachtet und deren Gesamtempfindlichkeit und Resonanzgüte bestimmt [13, 14]. Als nächstes wird eine lokale Funktionalisierung der Nanostrukturen anhand von Analysen des elektrischen Feldes innerhalb dieser Strukturen simulativ erforscht.

\section{Spektrale Analyse von multiperiodischen und aperiodischen Nanostrukturen}

Hier werden multiperiodische und aperiodische Gitterstrukturen in photonischen Kristallen zur Steigerung der Empfindlichkeit untersucht. Multiperiodische Strukturen werden durch die logische ODER- Überlagerung von zwei oder mehr monoperiodischen Gittern erzeugt, wie der Abb. 4 entnommen werden kann. 


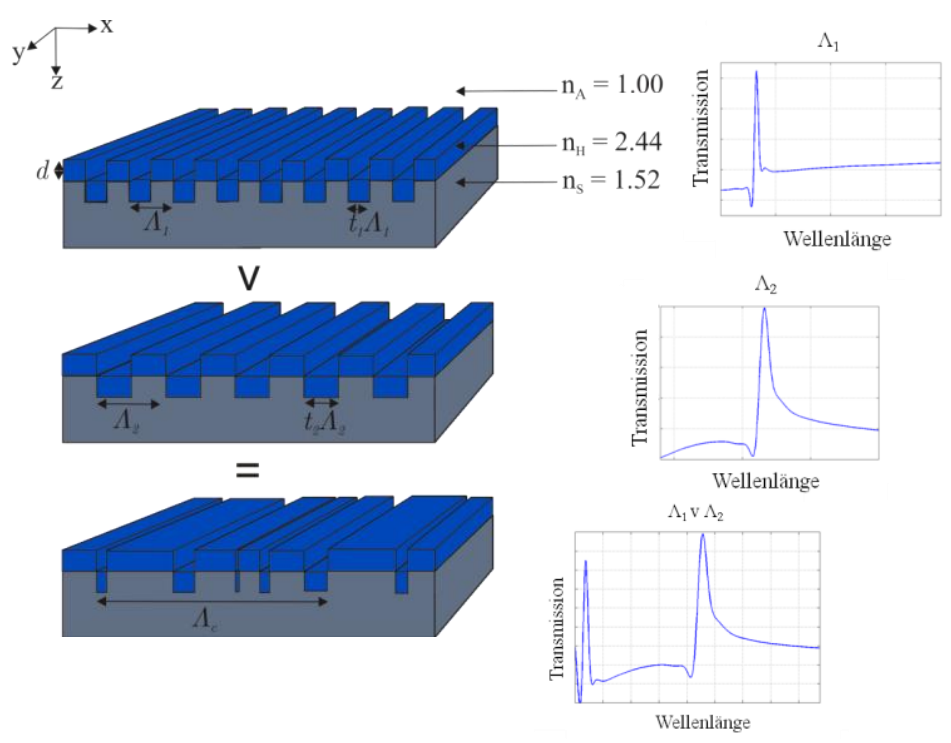

Abb. 4: Funktionsprinzip zur Erzeugung von multiperiodischen Gitterstrukturen (links) und die resultierenden Resonanzen zu den einzelnen Gitterstrukturen (rechts).

Dadurch weisen sie mehrere Resonanzen auf, deren zentrale Wellenlängen durch die Ausgangsgitterstrukturen vorgegeben sind. Durch die Variation von verschiedenen Designparametern, wie z.B. dem Tastverhältnis und der Anzahl der überlagerten Perioden, wird die Struktur mit der höchsten Empfindlichkeit verglichen zu der Empfindlichkeit der bisher verwendeten monoperiodischen photonischen Kristalle identifiziert. Hierzu wurden die spektralen Eigenschaften der Güte $Q=\lambda_{R} / F W H M\left(\lambda_{R}\right.$ : Resonanzwellenlänge; FWHM: Halbwertsbreite der Resonanz) und der Empfindlichkeit $S=\Delta \lambda / \Delta n(\Delta \lambda$ : Wellenlängenverschiebung der Resonanz; $\Delta n$ : Brechungsindexänderung) auf Brechungsindexänderungen von solchen multi- aber auch von aperiodischen Nanostrukturen simulativ und experimentell untersucht. Zur Realisierung von aperiodischen Nanostrukturen wurden exemplarisch binäre Sequenzen der drei mathematischen Folgen Rudin-Shapiro-Folge, Morse-Folge und Fibonacci-Folge in eindimensionale symmetrische Gitterstrukturen übersetzt. Dabei repräsentiert $A$ das Hochindexmaterial und $B$ das Substratmaterial (Tab. 1, Abb. 5).

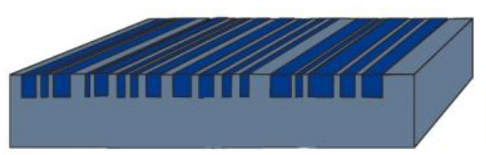

Rudin- Shapiro- Folge (RS)

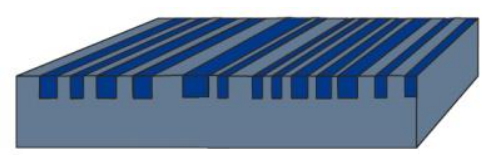

Morse- Folge (MF)

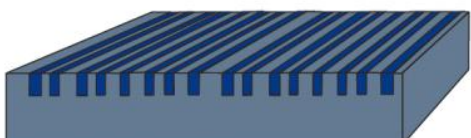

Fibonacci- Folge (F)

Abb. 5: Schemazeichnung der simulierten, aperiodischen Strukturen.

Für die Simulation der aperiodischen Gitterstrukturen wurde ein RCW (rigorous coupled wave)- Algorithmus in Matlab implementiert [15], während die multiperiodischen Gitterstrukturen anhand der Finite-Elemente-Methode (Programm: COMSOL) theoretisch betrachtet wurden [16]. In der folgenden Tabelle sind alle untersuchten Strukturen zusammengefasst.

Tab. 1: Untersuchte multiperiodische und aperiodische Strukturen mit Periode $\wedge$ und Tastverhältnis $t$

\begin{tabular}{lll}
\hline & Typ & Beschreibung \\
\hline$M$ & $\begin{array}{l}\text { Mono-periodisches } \\
\text { Gitter }\end{array}$ & $\Lambda=300 \mathrm{~nm} ; t=0,4$ \\
\hline
\end{tabular}




\begin{tabular}{|c|c|c|}
\hline M2 & $\begin{array}{l}\text { zweiperiodisches } \\
\text { Gitter }\end{array}$ & $\begin{array}{l}\Lambda_{1}=250 \mathrm{~nm} ; \Lambda_{2}=300 \mathrm{~nm} \\
t_{1}=0,3 ; t_{2}=0,4\end{array}$ \\
\hline M3 & dreiperiodisches Gitter & $\begin{array}{l}\Lambda_{1}=250 \mathrm{~nm} ; \Lambda_{2}=300 \mathrm{~nm} ; \Lambda_{3}=350 \mathrm{~nm} \\
t_{1}=0,3 ; t_{2}=0,4 ; t_{3}=0,3\end{array}$ \\
\hline M4 & vierperiodisches Gitter & $\begin{array}{l}\Lambda_{1}=250 \mathrm{~nm} ; \Lambda_{2}=300 \mathrm{~nm} ; \Lambda_{3}=350 \mathrm{~nm} ; \Lambda_{4}=400 \mathrm{~nm} \\
t_{1}=0,3 ; t_{2}=0,4 ; t_{3}=0,3 ; t_{4}=0,3\end{array}$ \\
\hline$R S$ & Rudin-Shapiro-Folge & $\begin{array}{l}\text { Substitution } A A \rightarrow A A A B, A B \rightarrow A A B A, B A \rightarrow B B A B, B B \rightarrow B B B A ; \\
\text { Bitlänge } h=50 \mathrm{~nm} ; N=128 \text { Serienkomponenten; } \\
\Lambda=6375 \mathrm{~nm} \text { Periode der Superzelle }\end{array}$ \\
\hline$M F$ & Morse-Folge & $\begin{array}{l}\text { Substitution } A \rightarrow A B, \mathrm{~B} \rightarrow \mathrm{BA} \text {; Bitlänge } h=50 \mathrm{~nm} ; N=128 \text { Serienkom- } \\
\text { ponenten; } \Lambda=12750 \mathrm{~nm} \text { Periode der Superzelle }\end{array}$ \\
\hline$F$ & Fibonacci-Folge & $\begin{array}{l}\text { Substitution } \mathrm{A} \rightarrow \mathrm{AB}, B \rightarrow A \text {; Bitlänge } h=50 \mathrm{~nm} ; N=144 \text { Serienkom- } \\
\text { ponenten; } \Lambda=14350 \mathrm{~nm} \text { Periode der Superzelle }\end{array}$ \\
\hline
\end{tabular}

Für die experimentellen Untersuchungen wurden die mono-, multi- und aperiodischen Strukturen mit Hilfe von UV Nanoprägelithographie von einem Master auf Glassubstrate repliziert und mit Niobpentoxid $\left(\mathrm{Nb}_{2} \mathrm{O}_{5}, n_{N b 2 O 5}=2,3\right)$ als Hochindexmaterial bedampft [10].

In Abbildung 6 sind die simulierten und experimentell ermittelten Spektren für das monoperiodische $M$ und die drei multiperiodischen Gitter (M2-M4) mit unterschiedlichen Analyten (Luft und Wasser) an der Oberfläche dargestellt. Zur besseren Vergleichbarkeit wurden die einzelnen Spektren auf ihren maximalen Intensitätswert normiert. Allgemein konnte festgestellt werden, dass zum einen die Brechungsindexerhöhung eine Intensitätsreduzierung der Resonanz in Wasser im Vergleich zu Luft hervorruft und dass zum anderen die Intensität der Resonanzen vom multiperiodischen Gitter im Vergleich zum monoperiodischen Ausgangsgitter deutlich schwächer ausfallen. Je mehr Perioden überlagert werden, je schwächer werden die Intensitäten der dominanten Resonanzen. Über die Variation der Tastgrade jedes Ausgangsgitters, also dem Verhältnis von Stegbreite zur Periode, kann allerdings die Intensität jeder einzelnen Resonanz gesteuert und angepasst werden [14].
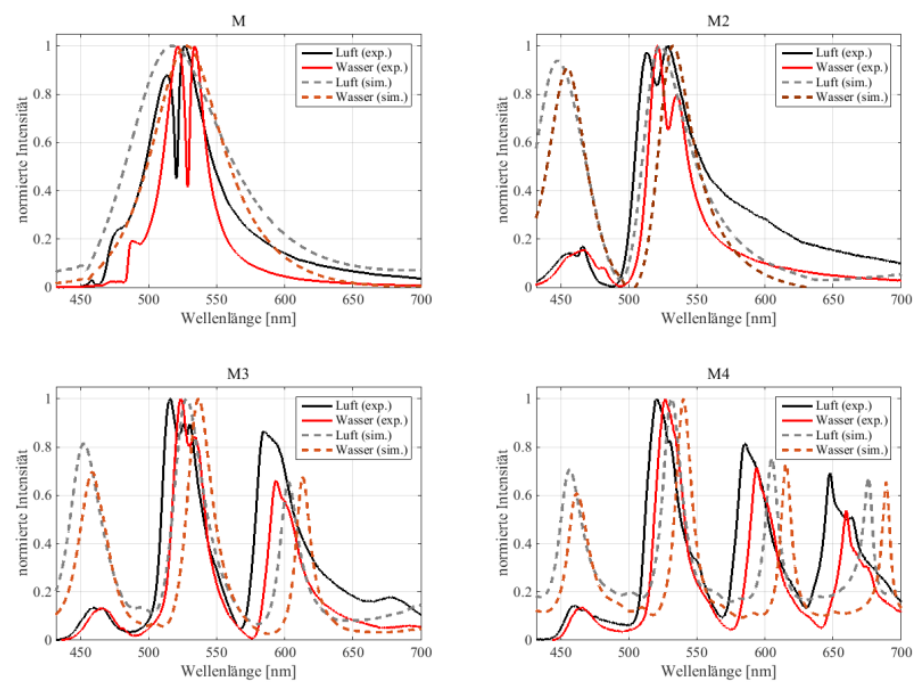

Abb. 6: Simulierte (sim.) und gemessene (exp.) Transmissionsspektren mit Luft $\left(n_{\text {Luft }}=1,00\right)$ und Wasser $\left(n_{\text {Wasser }}=1,33\right)$ als umgebendes Medium vom monoperiodischen $M$ (oben links) und den multiperiodischen Gitter $M 2$ (oben rechts), $M 3$ (unten links) und M4 (unten rechts).

Sowohl in den Simulationen als auch im Experiment sind für jede zusätzlich überlagerte Periode zusätzliche Resonanzphänomene im optischen Spektrum zu beobachten. Auch reagieren die Resonanzen aller Strukturen auf die Brechungsindexänderung $\Delta n$ von Luft zu Wasser als umgebendes Medium mit einer Verschiebung der Resonanzwellenlänge. In Tabelle 2 sind neben der Halbwertsbreite (FWHM) und der Resonanzpositionen $\left(\lambda_{\text {res }}\right)$ auch die Gesamtempfindlichkeit $S=\Delta \lambda / \Delta n$, also wie stark die Resonanzen $\Delta \lambda$ bedingt durch die Brechungsindexänderung von Luft zu Wasser als umgebendes Medium schieben, gegeben. Im Vergleich zu den simulierten Ergebnissen weisen die 
gemessenen Resonanzen speziell für die mono- und zweiperiodische Gitterstruktur Doppelpeaks auf. Diese entstehen durch die nicht perfekte Einstrahlung um $0^{\circ}$ im experimentellen Messaufbau. Durch die Lochblende vor der Mikroskoplampe, die als Anregungsquelle verwendet wird, treffen Lichtstrahlen auch unter einem Winkel von ca. $\pm 2^{\circ}$ auf den Kristall und verursachen diese Aufspaltung der Resonanzen. Weil aufgrund dieser Resonanzformen die exakte Resonanzwellenlänge nicht ermittelt werden kann, wird hier statt der Güte die Halbwertsbreite angegeben. In der Simulation hingegen werden die Resonanzen mit einer planaren Welle in einem Winkel von $0^{\circ}$ angeregt, wodurch zusätzliche Winkelanteile nicht auftreten und der Effekt der Peakaufspaltung hier nicht beobachtet werden kann.

Wie aus der Tabelle 1 und früheren Veröffentlichungen [15] entnommen werden kann, kann für multiperiodische, photonische Quasikristalle keine Empfindlichkeitssteigerung im Vergleich zu dem monoperiodischen Gitter festgestellt werden. Jedoch nimmt die Güte der Resonanzen in der Simulation mit der Anzahl der überlagerten Perioden zu, was für eine bildgebende Sensorik von Vorteil ist. Die experimentellen Ergebnisse können eine Verbesserung der Halbwertsbreite und damit der Resonanzgüte allerdings nur teilweise bestätigen. Die multiperiodischen Strukturen haben zum Teil Stegbreiten von nur ca. $10 \mathrm{~nm}$ und diese sind dünnen Stegbreiten sind problematisch im Abformungsprozess. Dies erklärt die Abweichungen zwischen Simulation und Experiment.

Tab. 2: Empfindlichkeit $S$ und Halbwertsbreite FWHM der simulierten (sim.) und gemessenen (exp.) mono- bzw. multiperiodischen Nanostrukturen mit $\lambda_{R}$ der zentralen Wellenlänge für TE polarisiertes Licht

\begin{tabular}{|c|c|c|c|c|c|}
\hline Typ & $\lambda_{R}[\mathrm{~nm}]$ & $\begin{array}{l}S(\operatorname{sim} .) \\
\text { [nm/RIU] }\end{array}$ & $\begin{array}{l}S \text { (exp.) } \\
\text { [nm/RIU] }\end{array}$ & $\begin{array}{l}\text { FWHM (sim.) } \\
\text { [nm] }\end{array}$ & $\begin{array}{l}\text { FWHM (exp.) } \\
{[\mathrm{nm}]}\end{array}$ \\
\hline $\bar{M}$ & 526 & 30,30 & 23,03 & 88,3 & 47,4 \\
\hline \multirow[t]{2}{*}{ M2 } & 455 & 21,21 & 30,30 & 50,0 & 28,2 \\
\hline & 529 & 30,03 & 17,87 & 45,1 & 49,1 \\
\hline \multirow[t]{3}{*}{ M3 } & 460 & 18,18 & 17,57 & 24,2 & 26,1 \\
\hline & 516 & 27,27 & 24,84 & 25,8 & 29,6 \\
\hline & 584 & 30,30 & 26,96 & 16,2 & 33,4 \\
\hline \multirow[t]{4}{*}{ M4 } & 460 & 15,15 & 16,66 & 14,5 & 28,5 \\
\hline & 520 & 27,27 & 18,78 & 17,7 & 26,2 \\
\hline & 586 & 33,33 & 22,12 & 11,3 & 28,5 \\
\hline & 648 & 39,39 & 34,54 & 11,2 & 33,4 \\
\hline
\end{tabular}
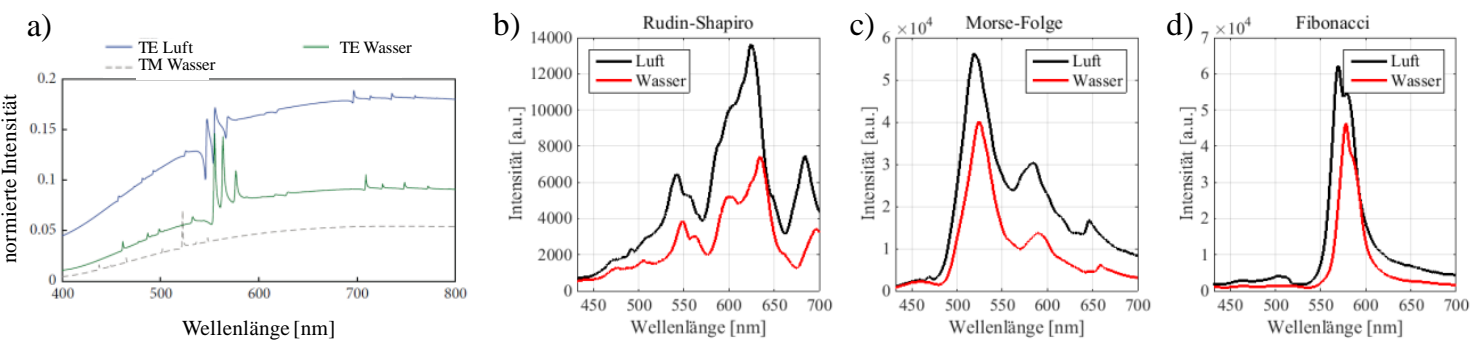

Abb. 7: (a) Simulierte Resonanzspektren der Fibonacci-Folge und (b-d) gemessene Resonanzspektren mit Luft $\left(n_{\text {Luft }}=1,00\right)$ und Wasser $\left(n_{\text {Wasser }}=1,33\right)$ als umgebendes Medium von photonischen Quasikristallen mit aperiodischen Nanostrukturen (RudinShapiro, Morse, Fibonacci).

Die aperiodischen Strukturen wurden gemäß der in Tabelle 1 dargestellten Substitutionsregel in eine binäre Sequenz und somit in eine eindimensionale Gitterstruktur übertragen. Die Substitutionsregeln wird dabei endlich oft ausgeführt, wodurch die Simulationszelle eine entsprechende endliche Größe erhält. So wurde die Anzahl der Iterationsschritte für die realisierten Gitterstrukturen auf N=6 für die Rudin-Shapiro-Folge, N=8 für die Morse-Folge und N=12 für die Fibonacci-Folge festgelegt. Im Vergleich zu den simulierten Ergebnissen von Neustock et al. [15] stimmen die in Abb. 7 b)- d) gezeigten Spektren nicht überein. Sie sind deutlich breiter und der Resonanzkamm, der durch die simulierte Fibonacci-Folge (Abb. 7 a)) erzeugt wurde, verschwimmt zu einer einzelnen Resonanz. Sowohl in der Simulation als auch im Experiment ergaben die aperiodischen Strukturen keine signifikante Empfindlichkeitssteigerung. Wegen der 
Resonanzformen kann die Halbwertsbreite für die gemessenen Resonanzen nicht bestimmt werden. Allerdings wurde auch bei diesen Strukturen in der Simulation die Güte der Resonanzen noch ein Mal deutlich erhöht, wie aus Tabelle 3 ersichtlich wird [15]. Allgemein zeigen beide Simulationsmethoden, dass die Resonanzgüte mit der Unordnung in den Strukturen steigt.

Tab. 3: Güte $Q$ und Empfindlichkeit S der simulierten (sim.) und gemessenen (exp.) aperiodischen Nanostrukturen mit $\lambda_{R}$ der zentralen Wellenlänge für TE polarisiertes Licht

\begin{tabular}{|c|c|c|c|c|c|}
\hline Тур & $\begin{array}{l}\lambda_{R}(\operatorname{sim} .) \\
{[\mathrm{nm}]}\end{array}$ & $\begin{array}{l}S \text { (sim.) } \\
\text { [nm/RIU] }\end{array}$ & $Q(\operatorname{sim})$. & $\begin{array}{l}\lambda_{R}(\exp ) \\
{[\mathrm{nm}]}\end{array}$ & $\begin{array}{l}S \text { (exp.) } \\
\text { [nm/RIU] }\end{array}$ \\
\hline \multirow[t]{5}{*}{$R S$} & 450 & 16 & 62 & 542,1 & 23,03 \\
\hline & 492 & 19 & 73 & 625,8 & 28,18 \\
\hline & 533 & 25 & 95 & 684,4 & 13,4 \\
\hline & 595 & 26 & 104 & - & - \\
\hline & 740 & 49 & 150 & - & - \\
\hline \multirow[t]{4}{*}{$M F$} & 418 & 19 & - & 519,8 & 15,75 \\
\hline & 530 & 18 & - & 585,9 & 19,69 \\
\hline & 544 & 20 & - & 646,1 & 37,57 \\
\hline & 657 & 33 & - & - & - \\
\hline \multirow[t]{2}{*}{$F$} & 515 & 23 & - & 569,7 & 28,66 \\
\hline & 557 & 24 & - & - & - \\
\hline
\end{tabular}

\section{Analyse des elektrischen Feldes innerhalb der Nanostrukturen}

Für die Proteindetektion mit nanostrukturierten Oberflächen ist die stochastische Verteilung der wenigen zu detektierenden Proteine über die gesamte Sensorfläche von Nachteil. Durch die hier betrachtete lokale Funktionalisierung könnten die Proteine gezielt an die Positionen mit der höchsten elektrischen Feldintensität angebunden werden und somit das höchste Signal erzeugen. Durch die Betrachtung der elektrischen Feldverteilung in den multiperiodischen und aperiodischen Strukturen wird das Potential zur Empfindlichkeitssteigerung hier nun theoretisch untersucht. Über die elektrische Feldverteilung in und oberhalb einer Struktur kann die Ausbildung der einzelnen Moden untersucht und somit über die Berechnung der elektrischen Feldintensität $|E|^{2}$ an der Oberfläche auf die Empfindlichkeit der Struktur geschlossen werden [17, 18]. Je höher die elektrische Feldintensität an der Oberfläche ist, desto empfindlicher reagiert die Struktur auf Brechungsindexänderungen. 

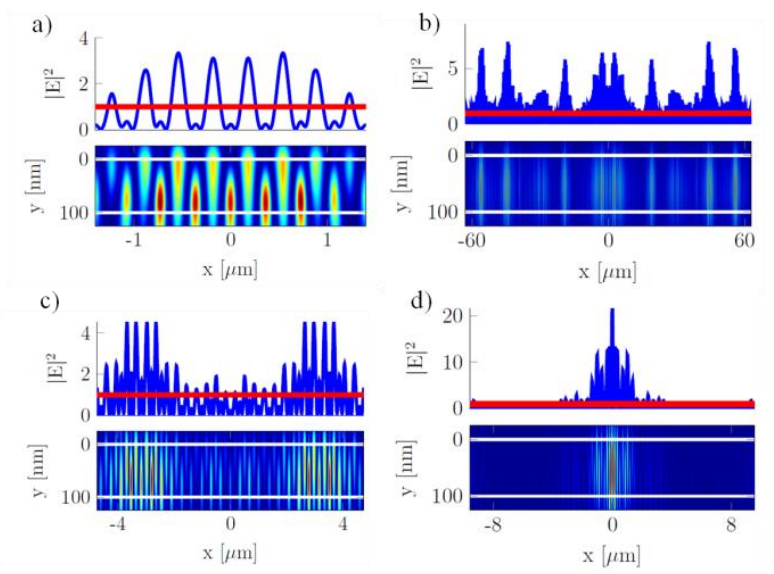

Abb. 8: Elektrische Feldintensität an der Oberfläche und elektrische Feldverteilung innerhalb der Strukturen: a) zweiperiodisches Gitter, b) fünfperiodisches Gitter, c) Rudin-Shapiro-Folge und d) Morse-Folge.Im unteren Bild ist jeweils ein Schnitt durch die Struktur gezeigt und die weißen Linien markieren die Grenzflächen des Wellenleiters zum Substrat und zum Messbereich.

Für Abbildung 8 wurde zunächst die elektrische Feldverteilung (jeweils unteres Bild) für jede Resonanz, die sich in den Strukturen ausbildet, mit Hilfe des RCWA-Algorithmus berechnet. Es sind sehr gut die evaneszenten Anteile der Moden sichtbar, die aus der Struktur herausragen und die photonischen Kristalle erst für die Sensorik nutzbar machen. Als nächstes wurde der Mittelwert der elektrischen Feldintensität über die gesamte Strukturoberfläche berechnet (rote Linie). Dies entspricht der Systemantwort des photonischen Kristalls bei einer flächigen Funktionalisierung des Sensors. Anschließend wurde die elektrische Feldintensität ortsaufgelöst in x-Richtung berechnet (blaue Linie). Wie zu erkennen ist, ist die mittlere elektrische Feldintensität für alle Strukturen ziemlich ähnlich, was auch bei der Betrachtung der Empfindlichkeiten zu erwarten war. Jedoch sind die Moden in den verschiedenen Strukturen unterschiedlich stark lokalisiert und die maximalen Feldintensitäten deutlich höher als der Mittelwert, was eine deutliche Empfindlichkeitssteigerung in diesen Bereichen verspricht. Z.B. liefert die Resonanz bei $\lambda_{R}=600 \mathrm{~nm}$ der Morse-Folge die stärkste lokalisierte Feldverteilung mit einer 20-fach höheren Feldintensität auf der Oberfläche als der Mittelwert (Abb. 8 d).

a)

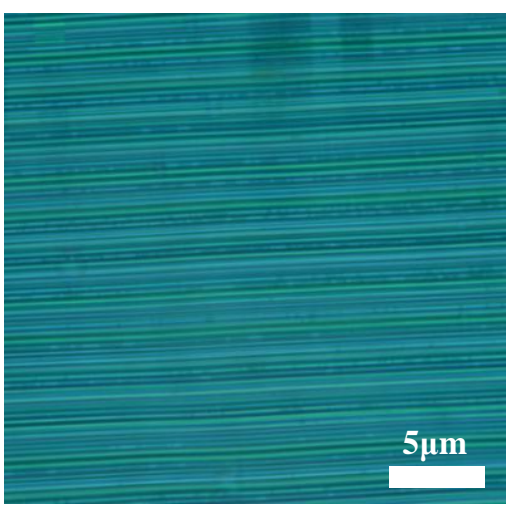

b)

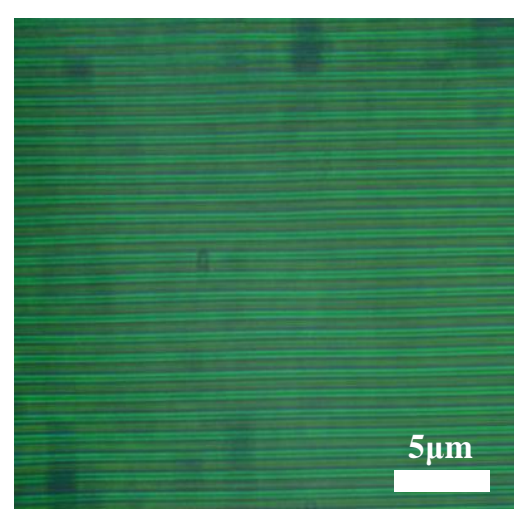

Abb. 9: Fotos von photonischen Quasikristallen: (a) vierperiodisches Gitter und (b) Morse-Folge als Gitterstruktur, aufgenommen mit gekreuzten linearen Polfiltern. 

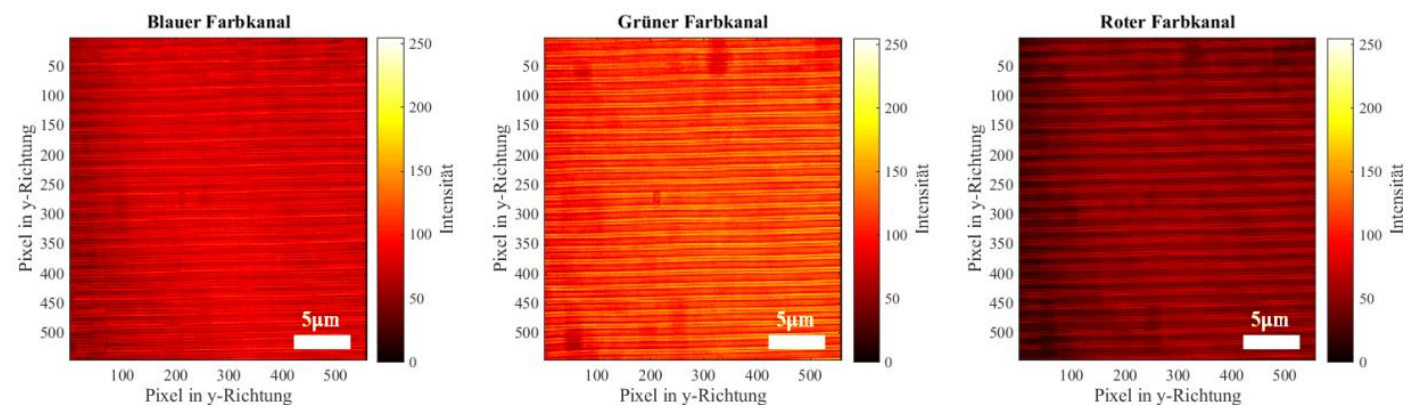

Abb. 10: Foto des photonischen Quasikristalls mit Morse-Folge wurde in seine Grundfarbwerte zerlegt. Gezeigt sind die daraus resultierenden Falschfarbenbilder des blauen (links), grünen (Mitte) und roten (rechts) Farbkanals.

Dieser Effekt wurde in ersten experimentellen Untersuchungen bestätigt, wie man in Abb. 9 sieht. Über Elektronenstrahllithographie wurden die vierperiodische Gitterstruktur und die Morse-Folge, wie oben beschrieben, in einen Nickel-Master geschrieben. Dann wurde die Struktur über das Prägeverfahren auf ein Glassubstrat übertragen und als Hochindexmaterial erneut $\mathrm{Nb}_{2} \mathrm{O}_{5}$ mit einer Schichtdicke von $85 \mathrm{~nm}$ aufgetragen. Für das Foto der so entstandenen photonischen Quasikristalle (Abb. 9) wurden die Kristalle jeweils auf einen Mikroskoptisch zwischen zwei gekreuzte, lineare Polfilter platziert und mit der Mikroskoplampe bestrahlt. Dadurch wird nahezu das ganze Anregungslicht unterdrückt und nur das Licht, welches mit den Moden in der Struktur interagiert, über ein Objektiv mit 40-facher Vergrößerung zum Kamerasensor geleitet [19]. Beide Fotos zeigen deutlich, dass die einzelnen Resonanzen sich nur örtlich entlang der Struktur ausbilden können. Durch die Betrachtung der blauen, grünen und roten Farbwerte des Fotos vom Quasikristall mit Morse-Folge (Abb. 9 rechts) wird dieser Eindruck bestätigt. Entsprechend zu den Simulationen wird auch hier die Ausbreitung der Resonanz im roten Spektralbereich sehr stark örtlich begrenzt (Abb. 10 rechts).

\section{Schlussfolgerung}

Wir demonstrierten hier eine Plattformtechnologie, die eine markerfreie und spezifische Detektion von mehreren Proteinen gleichzeitig über ein Kamera-basiertes Messsystem ermöglicht. Wir untersuchten die Gesamtempfindlichkeit und die Resonanzgüte von multiperiodisch und aperiodisch nanostrukturierten Wellenleitern, um das Detektionslimit unseres Systems zu verbessern. Mit einer Erhöhung der Unordnung in der Gitterstruktur, d.h. von streng monoperiodisch über multiperiodisch zu aperiodisch, konnte eine Verbesserung der Resonanzgüte beobachtet werden. Eine Steigerung der Gesamtempfindlichkeit bei diesen Strukturen konnte generell nicht festgestellt werden. Jedoch zeigt sich sowohl in den Simulationen als auch in den Experimenten eine starke Lokalisierung der Resonanzen , was mit einer lokalen Felderhöhung und somit mit einer potentiellen, lokalen Empfindlichkeitssteigerung einhergeht. Durch die Funktionalisierung dieser Bereiche mit den Liganden erscheint eine deutliche Verbesserung des Detektionslimits möglich.

\section{Danksagung}

Die Autoren bedanken sich beim European Research Council (ERC) für die finanzielle Unterstützung innerhalb des Projektes „PhotoSmart“ (307800). Auch bedanken wir uns bei Karlsruhe Nano and Micro Facility (KNMF, http://www.kit.edu/knmf) für die Unterstützung bei der Fertigung der multiperiodischen und aperiodischen Gitterstrukturen.

\section{Literaturverzeichnis}

[1] J. McCord, R. M. Nowak, P. A. McCullough, C. Foreback, S. Borzak, G. Tokarski, M. C. Tomlanovich, G. Jacobsen, W. D. Weaver, Circulation 104, 1483-1488 (2001); doi: 10.1161/hc3801.096336

[2] M. B. Schulze, K. Hoffmann, H. Boeing, J. Linseisen, S. Rohrmann, M. Möhlig, A. F. H. Pfeiffer, J. Spranger, C. Thamer, H.-U. Häring, A. Fritsche, H.-G. Joost, Diabetes Care 30, 510-515 (2007); doi: 10.2337/dc06-2089 
[3] F. Wei, P. Patel, W. Liao, K. Chaudhry, L. Zhang, M. Arellano-Garcia, S. Hu, D. Elashoff, H. Zhou, S. Shukla, F. Shah, C.M. Ho, D. T. Wong, Clinical Cancer Research 15, 4446-4452 (2009); doi: 10.1158/1078-0432.CCR-09-0050

[4] C.-S. Huang, V. Chaudhery, A. Pokhriyal, S. George, J. Polans, M. Lu, R. Tan, R. C. Zangar, B. T. Cunningham, Analytical Chemistry 84, 1126-1133 (2012); doi: 10.1021/ac202817q

[5] O. Bleher, A. Schindler, M.-X. Yin, A. B. Holmes, P. B. Luppa, G. Gauglitz, G. Proll, Analytical \& Bioanalytical Chemistry 406, 3305-3314 (2014); doi:10.1007/s00216-013-7504-y

[6] P. Kozma, F. Kehl, E. Ehrentreich-Förster, C. Stamm, and F. F. Bier, Biosensors and Bioelectronics 58, 287-307 (2014); doi:10.1016/j.bios.2014.02.049

[7] Y. Wang, D. Li, W. Ren, Z. Liu, S. Dong, E. Wang, Chemical Communications 22, 2520-2522, (2008); doi: 10.1039/B801055B

[8] J. A. Lee, S. Hwang, J. Kwak, S. I. Park, S. S. Lee, K.-C. Lee, Sensors and Actuators B 129, 372-379, (2008); doi:10.1016/j.snb.2007.08.034

[9] M. A. Lifson, D. B. Roy, B. L. Millers, Analytical Chemistry 86, 1016-1022, (2014); doi: 10.1021/ac401523e

[10] S. Jahns, M. Bräu, B. Meyer, T. Karrock, S. B. Gutekunst, L. Blohm, C. Selhuber-Unkel, R. Buhmann, Y. Nazirizadeh, M. Gerken, Biomedical Optics Express 6, 3724-3736 (2015); doi:10.1364/BOE.6.003724

[11] S. Jahns, S. B. Gutekunst, C. Selhuber-Unkel, Y. Nazirizadeh, M. Gerken, Microsystem Technologies, 1-6 (2015); doi: 10.1007/s00542-015-2746-6

[12] S. Jahns, Y. Nazirizadeh, B. Meyer, S. B. . Gutekunst, C. Selhuber-Unkel, M. Gerken, IEEE SENSORS, 1-3 (2013); doi: 10.1109/ICSENS.2013.6688384

[13] L. Dal Negro, S.V. Boriskina, Laser \& Photonics Reviews 6, 178-218 (2012); doi: 10.1002/lpor.201000046

[14] C. Kluge, J. Adam, N. Barie, P. J. Jakobs, M. Guttmann, M. Gerken, Optics Express 22, A1363-A1371 (2014); doi: 10.1364/OE.22.0A1363

[15] L. T. Neustock, S. Jahns, J. Adam, M. Gerken, Journal of Sensors 501, 6174527 (2016); doi: 10.1155/2016/6174527

[16] L. T. Neustock, M. Paulsen, S. Jahns, J. Adam, M. Gerken, Electromagnetics in Advanced Applications (ICEAA), 2016 International Conference on. IEEE, (2016). doi: 10.1109/ICEAA.2016.7731570

[16] I. D. Block, P. C. Mathias, S. I. Jones, L. O. Vodkin, B. T. Cunningham, Applied Optics 48, 6567-6574 (2009); doi: 10.1364/AO.48.006567

[17] Y. Ding and R. Magnusson, "Resonant leaky-mode spectralband engineering and device applications," Optics Express 12, 5661-5674 (2004); doi: 10.1364/OPEX.12.005661

[18] Y. Nazirizadeh, U. Bog, S. Sekula, T. Mappes, U. Lemmer, M. Gerken, Optics Express 18, 19120-19128 (2010); doi: 10.1364/OE.18.019120 